\title{
A Validated Liquid Chromatography-Mass Spectrometry Method for the Detection and Quantification of Oxidative Metabolites of 2,2',4,4'-Tetrabromodiphenyl Ether in Rat Hepatic Microsomes
}

\author{
Sarah Catherine Moffatt ${ }^{1}$, Patrick Robert Edwards ${ }^{2}$, András Szeitz ${ }^{1}$, Stelvio Mario Bandiera ${ }^{{ }^{*}}$ \\ ${ }^{1}$ Faculty of Pharmaceutical Sciences, The University of British Columbia, Vancouver, Canada \\ ${ }^{2}$ Faculty of Pharmacy, University of Toronto, Toronto, Canada \\ E-mail: sarah.moffatt@gmail.com, bandiera@interchange.ubc.ca \\ Received January 5, 2011; revised March 4, 2011; accepted March 15, 2011
}

\begin{abstract}
In the present study, we developed and validated an analytical method using ultra performance liquid chromatography-mass spectrometry (UPLC/MS) for the quantitative determination of 2,2',4,4'-tetrabromodiphenyl ether (BDE-47) metabolism by rat hepatic microsomes. BDE-47 is a brominated flame retardant that was widely used in a variety of consumer products and has subsequently been identified as a ubiquitous environmental contaminant. Hydroxy-bromodiphenyl ethers (OH-BDEs) were isolated from rat hepatic microsomes by liquid-liquid extraction. Chromatographic separation was achieved by UPLC on a $\mathrm{C}_{18}$ column with gradient elution using a mobile phase consisting of methanol and water, each containing $0.1 \%$ formic acid, at a flow rate of $0.2 \mathrm{~mL} / \mathrm{min}$. Detection and quantification were performed using a mass spectrometer in single ion recording mode with negative electrospray ionization. The UPLC/MS method was validated for linearity, limit of quantification (LOQ), accuracy, precision and recovery. The weighted calibration curves $\left(1 / \mathrm{X}^{2}\right)$ were linear over a concentration range of 5 - $250 \mathrm{nM}$ with LOQ values between $5 \mathrm{nM}$ and $50 \mathrm{nM}$ for the individual OH-BDEs. Intra- and inter- day accuracy (\%DEV) and precision (\%RSD) values ranged from $-11.7 \%$ to $9.5 \%$ and $5.9 \%$ to $16.5 \%$, respectively. Recovery values of $70 \%$ to $90 \%$ were obtained for all OH-BDEs. The validated method allowed us to successfully analyze metabolite formation following incubation of BDE-47 with hepatic microsomes prepared from phenobarbital-treated rats. Results demonstrate that the UPLC/MS method has sufficient sensitivity and reproducibility to fully characterize the in vitro metabolism of BDE-47 and possibly other PBDEs.
\end{abstract}

Keywords: BDE-47, Hepatic Metabolism, Polybrominated Diphenyl Ethers, Rat Hepatic Microsomes, Ultra Performance Liquid Chromatography-Mass Spectrometry.

\section{Introduction}

Polybrominated diphenyl ethers (PBDEs) are halogenated aromatic hydrocarbons that have been used as additive flame retardants on a variety of consumer products since 1965 [1]. PBDEs were marketed as commercial mixtures containing a limited number of the 209 possible brominated diphenyl ether (BDE) congeners [2]. The penta-BDE mixture, which was used extensively in North America [3], was composed predominantly of 2,2', 4,4'-tetrabromodiphenyl ether (BDE-47), 2,2',4,4',5-pentabromodiphenyl

ether

(BDE-99), 2,2',4,4',6-pentabromodiphenyl ether (BDE-100), 2,2',4,4',5,5'-hexabromo- diphenyl ether (BDE-153) and 2,2',4,4',5,6'-hexabromo- diphenyl ether (BDE-154) [4]. Penta-BDE was applied to epoxy resins, textiles, paints and flexible polyurethane foam, which was used in upholstered furniture, mattresses and carpet padding $[5,6]$. PBDEs are not chemically bound to the polymer components of the products to which they are applied and can be released into the environment during manufacture [5], use [7] and disposal [8] of these products. This factor, together with the high lipophilicity, chemical stability and the widespread use of BDE mixtures has resulted 
in the ubiquitous distribution of PBDEs in the environment [1]. BDE-47, for example, has been detected in air [9], sediment [10], fish [10,11], marine mammals [12,13] and in human blood [14], adipose tissue [15] and breast milk [16] and is frequently the predominant PBDE congener found in biotic samples [1]. Studies with laboratory animals have shown that developmental exposure to BDE-47 caused alterations in neuromotor activity [17] and exposure in utero produced changes to the reproductive system and thyroid gland of female rat pups [18].

Laboratory studies have shown that PBDEs can be metabolized by hepatic cytochrome P450 enzymes to hydroxy-BDEs (OH-BDEs) [19-22]. OH-BDEs are of toxicological interest as OH-BDEs show a greater affinity for the thyroid hormone receptor than the natural ligand or PBDEs themselves [19]. OH-BDEs have been detected in blood and feces of rodents treated with BDE-47 [21] and various OH-BDEs have been found in human plasma [23]. Several studies, including those mentioned above, examined the formation of OH-BDEs; however, the specific enzymes and enzyme kinetics of OH-BDEs formation are poorly understood. Characterization of BDE-47 metabolism in vitro is needed to develop a better understanding of the role of metabolism in the bioaccumulation and toxicity of BDE-47.

The most common method for the detection and quantification of PBDEs and OH-PBDEs in environmental samples has been gas chromatography-mass spectrometry (GC/MS) or gas chromatography coupled with electron capture detection (GC/ECD) [19]. In a study that examined the endocrine disrupting activity of BDEs following hepatic biotransformation, Hamers et al. [19] identified six hydroxylated metabolites of BDE-47 using a GC/MS method. GC-based methods are sensitive, but require derivatization of $\mathrm{OH}-\mathrm{BDEs}$, additional sample preparation time, the use of harmful derivatizing agents and possible underestimation of OH-BDE concentrations due to incomplete derivatization. Liquid chromatography coupled with mass spectrometry (LC/MS) provides an alternative analytical technique that does not require derivatization. Mas et al. demonstrated that LC/MS can be used to detect and quantify OH-BDEs in soil, fish, sludge and particulate matter that was spiked with a mixture of OH-BDEs [24]. However, their LC/MS method was not validated in a biological matrix [24] and its applicability to the detection and quantification of oxidative metabolites of BDE-47 generated in vitro or in vivo is unknown.

The aim of the present study was to develop and validate a UPLC/MS-based analytical method to detect and quantify OH-BDEs and apply this method to investigate the in vitro biotransformation of BDE-47 by rat hepatic microsomes.

\section{Materials and Methods}

\subsection{Chemicals and Reagents}

BDE-47 (neat, 99\% purity) was obtained from Chiron (Trondheim, Norway). 4'-Hydroxy-2,2',4-tribromodiphenyl ether (4'-OH-BDE-17), 2'-hydroxy-2,4,4'-tribromodiphenyl ether (2'-OH-BDE-28), 4-hydroxy-2,2',3,4'tetrabromodiphenyl ether (4-OH-BDE-42), 3-hydroxy-2, 2',4,4'-tetrabromodiphenyl ether (3-OH-BDE -47), 5-hydroxy-2,2',4,4'-tetrabromodiphenyl ether (5-OH-BDE47), 6-hydroxy-2,2',4,4'-tetrabromodiphenyl ether (6OH-BDE-47), 4'-hydroxy-2,2',4,5'-tetrabromodiphenyl ether (4'-OH-BDE-49) $(10 \mu \mathrm{g} / \mathrm{mL}$ or $50 \mu \mathrm{g} / \mathrm{mL}$ in acetonitrile, purity of at least 98\%) and 4'-hydroxy-2,2',4,6'tetrachlorobiphenyl (4'-OH-CB-50, neat, 99\% purity) were purchased from AccuStandard (New Haven, Connecticut, USA). Magnesium chloride, sucrose and nicotinamide adenine dinucleotide phosphate (NADPH) were purchased from Sigma-Aldrich (Oakville, Ontario, Canada). Methanol, methyl-tert-butyl ether, hexane, isopropanol, sodium hydroxide, hydrochloric acid and monoand di-basic potassium phosphate were purchased from Fisher Scientific (Ottawa, Ontario, Canada). Hydrochloric acid and all organic solvents were HPLC- grade or higher. Ultra pure water was obtained using a Milli-Q Synthesis system (Millipore, Billerica, MA, USA).

\subsection{Rat Hepatic Microsomes}

Adult male Long Evans rats (body weight between 160190 g) were purchased from Charles River Laboratories (Montreal, PQ, Canada). Rats were cared for in accordance with the principles and guidelines outlined by the Canadian Council of Animal Care. Rats $(n=6)$ were treated with sodium phenobarbital (PB, $80 \mathrm{mg} / \mathrm{kg} /$ day) for 3 days, as previously described by Edwards et al. [25]. Pooled hepatic microsomes were prepared by differential centrifugation as previously described [26]. Hepatic microsomes were aliquoted and stored at $-80^{\circ} \mathrm{C}$ until use. Protein concentration was determined by the method of Lowry, et al. with bovine serum albumin as the standard [27].

\subsection{Standard Solutions}

A stock solution of BDE-47 at a concentration of 2.5 $\mathrm{mM}$ was prepared in methanol. A stock solution of $\mathrm{OH}-\mathrm{BDE}$ standards containing 4'-OH-BDE-17, 2'-OH-BDE-28, 4-OH-BDE-42, 3-OH-BDE-47, 5-OH-BDE-47, 6- OH-BDE-47 and 4'-OH-BDE-49 (at $1.25 \mu \mathrm{M}$ each) was prepared in methanol. A second 
<smiles>Oc1ccc(Oc2ccc(Br)cc2Br)c(Br)c1</smiles>

Figure 1. Chemical structures of BDE-47 and seven possible hydroxy-metabolites.

stock solution of OH-BDE standards at $0.125 \mu \mathrm{M}$ each was prepared by diluting an aliquot of the first stock solution 10-fold in methanol. A stock solution of internal standard containing 4'-OH-CB-50 at a concentration of $125 \mu \mathrm{M}$ was prepared in methanol. Three quality control (QC) solutions containing OH-BDE standards at concentrations of 7.5, 37.5 and $175 \mathrm{nM}$ each and internal standard at a concentration of $1.25 \mu \mathrm{M}$ were prepared in methanol. These QC solutions were used for system suitability tests and recovery determination. All stock solutions were stored in amber vials at $-20^{\circ} \mathrm{C}$ until needed and each vial was vigorously vortex-mixed before use.

\subsection{Sample Preparation}

Stock solutions of OH-BDE standards were spiked into rat hepatic microsomes to prepare calibration standards (CS) samples for the generation of calibration curves. CS samples were prepared by mixing $1 \mathrm{mg}$ of hepatic microsomal protein, $50 \mathrm{mM}$ potassium phosphate buffer containing $3 \mathrm{mM}$ magnesium chloride $(\mathrm{pH}$ 7.4) and an appropriate volume of $\mathrm{OH}-\mathrm{BDE}$ stock solution in a final volume of $1 \mathrm{~mL}$. Final concentrations of the OH-BDE standards in the CS samples were 2.5, 5, 10, 25, 50, 100 and $250 \mathrm{nM}$. QC samples at three concentrations were prepared in the same manner. Final concentrations of OH-BDE standards in the QC samples were 7.5, 37.5 and $175 \mathrm{nM}$ (low, medium and high, respectively). The QC samples were used for the determination of accuracy and precision. Blank samples containing only hepatic microsomal protein and phosphate buffer were also prepared.

CS, QC and blank samples were incubated for $5 \mathrm{~min}$ at $37^{\circ} \mathrm{C}$ in a shaking water bath. Following incubation, 1 $\mathrm{mL}$ of ice-cold $0.5 \mathrm{M}$ sodium hydroxide was added, and each tube was immediately vortex-mixed. An aliquot of internal standard $(10 \mu \mathrm{L}$ of $125 \mu \mathrm{M})$ was added to each tube. Tubes were capped and placed in a $70^{\circ} \mathrm{C}$ water bath for $10 \mathrm{~min}$. After cooling to room temperature, 2 $\mathrm{mL}$ of $6 \mathrm{M}$ hydrochloic acid and $1 \mathrm{~mL}$ of isopropanol 
was added to each tube. Tubes were then vigorously vortex-mixed for $1 \mathrm{~min}$. Two $\mathrm{mL}$ of a methyl-tert-butyl ether: hexane $(1: 1 \mathrm{v} / \mathrm{v})$ mixture was then added to each
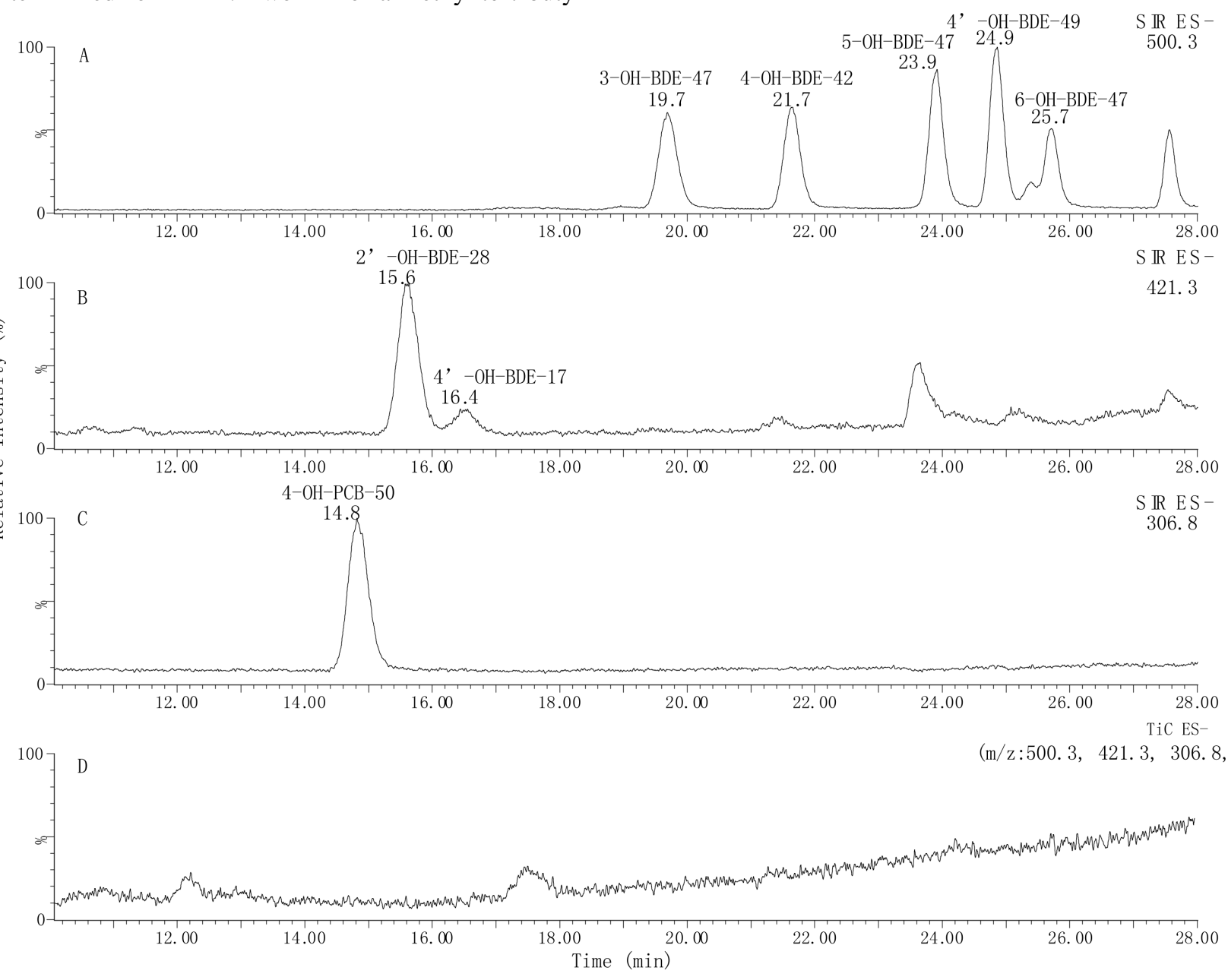

Figure 2. Representative UPLC/MS ion chromatograms showing peaks obtained by spiking rat hepatic microsomes (at a concentration of $1 \mathrm{mg} / \mathrm{mL}$ ) with a calibration standard stock solution (final concentration of OH-BDE standards was $50 \mathrm{nM}$ ) and the internal standard solution (final concentration was $1.25 \mu \mathrm{M}$. (a) Chromatogram of OH-tetra BDEs standards (3-OH-BDE-47, 4-OH-BDE-42, 5-OH-BDE-47, 4'-OH-BDE-49 and 6-OH-BDE-47) at $\mathrm{m} / \mathrm{z} 500.3$; (b) chromatogram of OH-tri-BDE standards (2'-OH-BDE-28 and 4'-OH-BDE-17) at $\mathrm{m} / \mathrm{z} 421.3$; (c) chromatogram of internal standard (4-OH-PCB-50) at $\mathrm{m} / \mathrm{z}$ 306.8; (d) total ion current of a blank sample.

tube. Tubes were vigorously vortex-mixed for 1 min and spun in a centrifuge at 2,500 rpm for $5 \mathrm{~min}$. The top organic layer was carefully removed and transferred into clean test tubes. The extraction procedure was repeated two more times. The organic phase from each extraction of the same sample was pooled and evaporated under a gentle flow of nitrogen. The residue was reconstituted in $250 \mu \mathrm{L}$ of methanol, vortex-mixed and filtered through a syringe filter (polytetrafluoroethylene membrane, 0.45 $\mu \mathrm{M}$ ) into a $300-\mu \mathrm{L}$ HPLC vial.

\subsection{UPLC/MS Conditions}

The UPLC/MS system consisted of a Waters Acquity
UPLC Sample Manager and a Waters Acquity UPLC Binary Solvent Manger connected to a Waters Quattro Premier XE triple quadrupole mass spectrometer equipped with a combined Electrospray Ionization (ESI) and Atmospheric Pressure Chemical Ionization (APCI) probe (Waters, Milford, MA, USA). Chromatographic separation was achieved using a Waters Acquity UPLC $\mathrm{BEH} \mathrm{C}_{18}(2.1 \times 100 \mathrm{~mm}, 1.7 \mu \mathrm{m})$ column, which was maintained at $50^{\circ} \mathrm{C}$. The autosampler tray was maintained at $10^{\circ} \mathrm{C}$ and the injection volume was $5 \mu \mathrm{L}$. The mobile phase consisted of water containing $0.1 \%(\mathrm{v} / \mathrm{v})$ formic acid (solvent $\mathrm{A}$ ) and methanol containing $0.1 \%$ $(v / v)$ formic acid (solvent B). Mobile phase solvents were filtered through a membraine filter (Millipore Durapore 
Membrane Filters, $0.22 \mu \mathrm{m} \mathrm{GV}$, Billerica, USA) prior to use. The gradient program was 35\% solvent $\mathrm{A}$ and $65 \%$ solvent B from 0 to 15 min followed by a linear increase to $80 \%$ solvent B from 15 to $30 \mathrm{~min}$. At $30.1 \mathrm{~min}$, sol-

Table 1. Limit of quantification (LOQ) of individual OH-BDE standards.

\begin{tabular}{|c|c|c|c|c|c|}
\hline Authentic Standard & LOQ (nM) & $\begin{array}{l}\text { Mean Measured Concentration } \\
\text { (nM) }\end{array}$ & Accuracy (\%Dev) & Precision (\%RSD) & $\mathrm{S} / \mathrm{N}$ \\
\hline 4'-OH-BDE-17 & 50 & $42.9 \pm 6.5$ & -14.2 & 15.1 & 5.4 \\
\hline 2'-OH-BDE-28 & 5 & $5.5 \pm 0.6$ & 10.0 & 10.9 & 4.9 \\
\hline 4-OH-BDE-42 & 10 & $11.3 \pm 0.9$ & 13.0 & 8.0 & 4.9 \\
\hline 3-OH-BDE-47 & 10 & $10.7 \pm 1.2$ & 7.0 & 11.2 & 4.9 \\
\hline 5-OH-BDE-47 & 5 & $5.6 \pm 0.3$ & 12.0 & 5.4 & 3.5 \\
\hline 6-OH-BDE-47 & 10 & $10.8 \pm 1.9$ & 8.0 & 17.5 & 4.1 \\
\hline 4'-OH-BDE-49 & 5 & $5.6 \pm 0.4$ & 12.0 & 7.1 & 3.9 \\
\hline
\end{tabular}

$\mathrm{n}=6$, mean $\pm \mathrm{SD}$

vent B was increased to $100 \%$ and maintained for 5 min. The column was then re-equilibrated with $35 \%$ solvent $\mathrm{A}$ and $65 \%$ solvent $B$ for $5 \mathrm{~min}$. The flow rate was maintained at $0.2 \mathrm{~mL} / \mathrm{min}$ and the total analysis time was 40 min. To protect the mass spectrometer from contamination, the mobile phase flow was diverted to waste between 0 and $10 \mathrm{~min}$ and 30 and $40 \mathrm{~min}$ of each injection.

The mass spectrometer was operated in negative electrospray ionization mode (ESI) using selected ion recording (SIR) at a capillary voltage of $3 \mathrm{kV}$, cone voltage of $40 \mathrm{~V}$, source temperature of $120^{\circ} \mathrm{C}$, desolvation temperature of $400^{\circ} \mathrm{C}$ and desolvation gas flow of $1005 \mathrm{~L} / \mathrm{h}$. The UPLC/MS system was controlled by MassLynx v. 4.1 software and Windows XP operating system. Chromatographic peaks corresponding to the OH-BDEs and the internal standard were identified by comparing the mass-to-charge ratio $(\mathrm{m} / \mathrm{z})$ and retention time values with those of the authentic standards: $\mathrm{m} / \mathrm{z} 421.3$ for 4 '-OHBDE-17 and 2'-OH-BDE-28; m/z 500.6 for 4-OH-BDE42, 3-OH-BDE-47, 5-OH-BDE-47, 6-OH-BDE-47 and 4'-OH-BDE-49; and $\mathrm{m} / \mathrm{z} 306.7$ for 4'-OH-CB-50.

\subsection{Method Validation}

The UPLC/MS method was validated for accuracy, precision, linearity, limit of quantification (LOQ), selectivity and recovery. Accuracy and precision were assessed using the three QC samples. Accuracy was calculated using the mean measured concentration and expressed as percent deviation (\%Dev) of the nominal concentration. Precision was expressed as percent relative standard deviation (\%RSD). To determine intra-day accuracy and precision, six replicates of each QC sample were prepared and analyzed on the same day. To determine inter-day accuracy and precision, QC samples were prepared and analyzed in triplicate on three separate days. The acceptance criteria for inter- and intra- day accuracy and precision were $\%$ Dev $\pm 20 \%$ and $\%$ RSD $\leq 20 \%$, respectively, for the low QC samples and \%Dev $\pm 15 \%$ and $\%$ RSD $\leq 15 \%$, respectively, for the medium and high QC samples.

Linearity of the calibration curve was assessed using the coefficient of determination $\left(\mathrm{R}^{2}\right)$. Calibration curves were generated for each $\mathrm{OH}-\mathrm{BDE}$ standard by plotting peak area ratios for each OH-BDE standard and internal standard (y-axis) against the corresponding nominal concentration of the OH-BDE standard (nM, X-axis) using linear regression analysis. To increase accuracy at the lower end of the calibration curve, a $1 / \mathrm{X}^{2}$ weighting factor was used. The acceptance criterion for linearity was $\mathrm{R}^{2} \geq 0.9$.

The LOQ of each OH-BDE standard was determined by preparing five replicates of CS samples between 2.5 and $50 \mathrm{nM}$ and a calibration curve. The LOQ was set as the lowest CS concentration that had a signal-to-noise $(\mathrm{S} / \mathrm{N})$ ratio at least three times higher than that of the 
blank sample with a $\pm 20 \%$ Dev and $\leq 20 \%$ RSD. The $\mathrm{S} / \mathrm{N}$ ratio was determined with MassLynx using the peak-to-peak method.

Selectivity was assessed by visually comparing the

Table 2. Intra-day accuracy (\%Dev) and precision (\%RSD).

\begin{tabular}{|c|c|c|c|c|}
\hline Metabolite & Nominal Concentration (nM) & Mean Measured Concentration (nM) & Accuracy (\%Dev) & Precision (\%RSD) \\
\hline \multirow{3}{*}{ 4'-OH-BDE-17 } & QC-Low (7.5) & $<\mathrm{LOQ}$ & n. d. & n. d. \\
\hline & QC-Med (37.5) & $<\mathrm{LOQ}$ & n. d. & n. d. \\
\hline & QC-High (175) & $167.0 \pm 17.7$ & -4.6 & 10.6 \\
\hline \multirow{3}{*}{ 2'-OH-BDE-28 } & QC-Low (7.5) & n. d. & n. d. & n. d. \\
\hline & QC-Med (37.5) & $36.1 \pm 3.3$ & -3.7 & 9.1 \\
\hline & QC-High (175) & $162.3 \pm 15.7$ & -7.3 & 9.7 \\
\hline \multirow{3}{*}{ 4-OH-BDE-42 } & QC-Low (7.5) & $<\mathrm{LOQ}$ & n. d. & n. d. \\
\hline & QC-Med (37.5) & $36.7 \pm 2.2$ & -2.1 & 5.9 \\
\hline & QC-High (175) & $153.9 \pm 15.4$ & -12.1 & 10.0 \\
\hline \multirow{3}{*}{ 3-OH-BDE-47 } & QC-Low (7.5) & $<\mathrm{LOQ}$ & n. d. & n. d. \\
\hline & QC-Med (37.5) & $36.8 \pm 2.8$ & -2.1 & 7.6 \\
\hline & QC-High (175) & $156.2 \pm 14.9$ & -10.7 & 9.5 \\
\hline \multirow{3}{*}{ 5-OH-BDE-47 } & QC-Low (7.5) & $8.2 \pm 1.4$ & 9.3 & 17.0 \\
\hline & QC-Med (37.5) & $36.6 \pm 3.0$ & -2.4 & 8.2 \\
\hline & QC-High (175) & $155.7 \pm 15.3$ & -11.0 & 9.8 \\
\hline \multirow{3}{*}{ 6-OH-BDE-47 } & QC-Low (7.5) & $<\mathrm{LOQ}$ & n. d. & n. d. \\
\hline & QC-Med (37.5) & $38.3 \pm 3.0$ & 2.1 & 7.8 \\
\hline & QC-High (175) & $178.2 \pm 19.1$ & 1.8 & 10.7 \\
\hline \multirow{3}{*}{ 4'-OH-BDE-49 } & QC-Low (7.5) & $7.8 \pm 0.5$ & 4.0 & 6.4 \\
\hline & QC-Med (37.5) & $35.8 \pm 3.2$ & -4.5 & 8.9 \\
\hline & QC-High (175) & $157.3 \pm 13.9$ & -10.1 & 8.8 \\
\hline
\end{tabular}

$<$ LOQ = Below limit of quantification; $\mathrm{n}$.d., not determined because metabolite concentration was below the LOQ; $\mathrm{n}=6$, mean \pm SD.

chromatograms obtained from blank samples with chromatograms from spiked CS samples at the LOQ value of each OH-BDE standard. Chromatograms were examined for the presence of interfering peaks with retention times that overlapped those of OH-BDE standards or the internal standard.

Recovery rates were determined using QC solutions and QC samples. Recovery was calculated by comparing the peak area of each OH-BDE standard in the QC sample with that in the QC solution at the same concentration.

\subsection{In Vitro Biotransformation of BDE-47}

Following validation, the UPLC/MS method was applied to investigate the in vitro biotransformation of BDE-47. Formation of hydroxy BDE-47 metabolites by rat hepatic microsomes was determined using a reaction mixture containing $50 \mathrm{mM}$ phosphate buffer, $0-2 \mathrm{mg} / \mathrm{mL}$ of rat hepatic mirosomal protein and $50 \mu \mathrm{M}$ BDE-47 $(20 \mu \mathrm{L}$ of $2.5 \mathrm{mM}$ in methanol) in a final volume of $0.99 \mathrm{~mL}$. After a 5-min pre-incubation at room temperature, the reaction was initiated by addition of $10 \mu \mathrm{L}$ of $100 \mathrm{mM}$ NADPH 
(final concentration $1 \mathrm{mM}$ ) and allowed to proceed at $37^{\circ} \mathrm{C}$ in a shaking water bath for 0 to $30 \mathrm{~min}$. The reaction was terminated by the addition of $1 \mathrm{~mL}$ of ice-cold $0.5 \mathrm{M}$ sodium hydroxide. Extraction and quantification

Table 3. Inter-day accuracy (\%Dev) and precision (\%RSD). of the OH-BDE metabolites was performed as described above. Samples were prepared in duplicate for each as-

Nominal Concentration (nM)

Mean Measured Concentration (nM)

Accuracy $(\% \mathrm{Dev})$

Precision (\%RSD)

\begin{tabular}{|c|c|c|c|c|}
\hline \multirow{3}{*}{ 4'-OH-BDE-17 } & QC-Low (7.5) & $<\mathrm{LOQ}$ & n. d. & n. d. \\
\hline & QC-Med (37.5) & $<\mathrm{LOQ}$ & n. d. & n. d. \\
\hline & QC-High (175) & $164.3 \pm 22.3$ & -6.1 & 13.6 \\
\hline \multirow{3}{*}{ 2'-OH-BDE-28 } & QC-Low (7.5) & n. d. & n. d. & n. d. \\
\hline & QC-Med (37.5) & $37.3 \pm 4.8$ & -0.4 & 12.7 \\
\hline & QC-High (175) & $158.4 \pm 12.0$ & -9.5 & 7.6 \\
\hline \multirow{3}{*}{ 4-OH-BDE-42 } & QC-Low (7.5) & $<\mathrm{LOQ}$ & n. d. & n. d. \\
\hline & QC-Med (37.5) & $37.8 \pm 4.0$ & 0.8 & 10.6 \\
\hline & QC-High (175) & $155.4 \pm 11.5$ & -11.2 & 7.4 \\
\hline \multirow{3}{*}{ 3-OH-BDE-47 } & QC-Low (7.5) & $<\mathrm{LOQ}$ & n. d. & n. d. \\
\hline & QC-Med (37.5) & $37.6 \pm 3.0$ & 0.3 & 10.1 \\
\hline & QC-High (175) & $155.2 \pm 11.5$ & -11.3 & 7.4 \\
\hline \multirow{3}{*}{ 5-OH-BDE-47 } & QC-Low (7.5) & $6.9 \pm 2.5$ & -8.0 & 8.0 \\
\hline & QC-Med (37.5) & $37.4 \pm 4.2$ & -0.3 & 11.3 \\
\hline & QC-High (175) & $156.3 \pm 13.0$ & -10.7 & 8.3 \\
\hline \multirow{3}{*}{ 6-OH-BDE-47 } & QC-Low (7.5) & $<\mathrm{LOQ}$ & n. d. & n. d. \\
\hline & QC-Med (37.5) & $39.5 \pm 4.3$ & 5.3 & 10.9 \\
\hline & QC-High (175) & $182.8 \pm 14.8$ & 4.4 & 8.1 \\
\hline \multirow{3}{*}{ 4'-OH-BDE-49 } & QC-Low (7.5) & $7.7 \pm 0.5$ & 2.7 & 6.5 \\
\hline & QC-Med (37.5) & $35.7 \pm 4.8$ & -4.8 & 13.4 \\
\hline & QC-High (175) & $154.6 \pm 11.0$ & -11.7 & 7.1 \\
\hline
\end{tabular}

say and experiments were performed 3 times on separate days. Blank samples contained only rat hepatic microsomes and buffer. Negative control samples did not contain BDE-47, NADPH, or rat hepatic microsomes. QC samples were included in each experiment to assess method performance.

\section{Results and Discussion}

\subsection{Optimization of UPLC/MS Parameters}

APCI and ESI in negative and positive modes were compared for the detection of OH-BDE standards by mass spectrometry using SIR. OH-BDE standards were not detected in positive ion mode. ESI operated in negative electrospray ion mode produced larger peak area counts than APCI. BDE-47 was not detected with APCI 
or ESI in either positive or negative ion mode. Flow injection analysis was used to determine the molecular ions $[\mathrm{M}-\mathrm{H}]^{-}$and optimal cone voltage $(40 \mathrm{~V})$ for all $\mathrm{OH}-$ BDE standards. A source temperature of $120^{\circ} \mathrm{C}$, a desol

Table 4. Recovery values of individual OH-BDE standards.

\begin{tabular}{|c|c|c|c|}
\hline Authentic Standard & Retention Time (min) & Nominal Concentration (nM) & Mean Recovery (\%) \\
\hline \multirow{3}{*}{ 4'-OH-BDE-17 } & \multirow{3}{*}{16.4} & QC-Low (7.5) & n. d. \\
\hline & & QC-Med (37.5) & n. d. \\
\hline & & QC-High (175) & $69.9 \pm 5.6$ \\
\hline \multirow{3}{*}{ 2'-OH-BDE-28 } & \multirow{3}{*}{15.6} & QC-Low (7.5) & n. d. \\
\hline & & QC-Med (37.5) & $86.7 \pm 5.3$ \\
\hline & & QC-High (175) & $72.3 \pm 4.3$ \\
\hline \multirow{3}{*}{ 4-OH-BDE-42 } & \multirow{3}{*}{21.7} & QC-Low (7.5) & \\
\hline & & QC-Med (37.5) & $84.0 \pm 6.0$ \\
\hline & & QC-High (175) & $74.0 \pm 5.3$ \\
\hline \multirow{3}{*}{ 3-OH-BDE-47 } & \multirow{3}{*}{19.7} & QC-Low (7.5) & n. d. \\
\hline & & QC-Med (37.5) & $88.0 \pm 4.4$ \\
\hline & & QC-High (175) & $76.7 \pm 5.0$ \\
\hline \multirow{3}{*}{ 5-OH-BDE-47 } & \multirow{3}{*}{23.4} & QC-Low (7.5) & $89.2 \pm 6.2$ \\
\hline & & QC-Med (37.5) & $88.2 \pm 4.6$ \\
\hline & & QC-High (175) & $75.2 \pm 4.7$ \\
\hline \multirow{3}{*}{ 6-OH-BDE-47 } & \multirow{3}{*}{25.7} & QC-Low (7.5) & n. d. \\
\hline & & QC-Med (37.5) & $88.2 \pm 4.3$ \\
\hline & & QC-High (175) & $74.0 \pm 4.9$ \\
\hline \multirow{3}{*}{ 4'-OH-BDE-49 } & \multirow{3}{*}{24.9} & QC-Low (7.5) & $87.6 \pm 5.3$ \\
\hline & & QC-Med (37.5) & $87.6 \pm 4.2$ \\
\hline & & QC-High (175) & $75.4 \pm 5.1$ \\
\hline
\end{tabular}

n. d., not determined because metabolite concentration was below the LOQ; $n=6$, mean \pm SD

(MRM) was also assessed. Product ion scans of the molecular ions were performed at different collision energy values. The main product ion was a bromine fragment $(\mathrm{m} / \mathrm{z} 79$ and $\mathrm{m} / \mathrm{z}$ 81). However, the sensitivity of the MRM method was much lower than that of the SIR method. Therefore, SIR was used for subsequent analyses.

Separation of the seven OH-BDE standards was achieved using a Waters Acquity UPLC BEH C 18 (2.1 × $100 \mathrm{~mm}, 1.7 \mu \mathrm{m})$ column and gradient elution. Several mobile phase combinations were tested, including mixtures of water and acetonitrile or water and methanol. A vation temperature of $400^{\circ} \mathrm{C}$ and a desolvation gas flow of $1005 \mathrm{~L} / \mathrm{H}$ were found to be optimal for the detection of $\mathrm{OH}-\mathrm{BDE}$ standards. Multiple reaction monitoring 
and run times were tried but complete baseline separation of 6-OH-BDE-47 and 4'-OH-BDE-49 was not achieved. In comparison, Mas, et al., [25] used a ternary mixture of ammonium acetate, acetonitrile and methanol, gradient elution and a shorter run time (20 min compared to $40 \mathrm{~min}$ in the present study) to resolve eight $\mathrm{OH}-$ BDEs but did not attain baseline separation of $5-\mathrm{OH}-$ BDE-47, 6-OH-BDE-47 and 4'-OH-BDE-49 or of three hydroxylated tribrominated diphenyl ethers.

\subsection{Optimization of Sample Preparation}

To achieve efficient extraction of the OH-BDE standards from the biological matrix (i.e., rat hepatic microsomes) and to minimize the possibility of unknown peaks that could interfere with the peaks of interest, variations of the sample preparation protocol were evaluated. Hexane, acetone, dichloromethane, methyl-tert-butyl ether, as well as, different ratios of acetone and hexane and of methyl-tert-butyl ether and hexane were tested as possible extraction solvents. Three extractions with a mixture of methyl-tert-butyl ether: hexane $(1: 1 \mathrm{v} / \mathrm{v})$ yielded the highest recovery of the OH-BDE standards (Table 4). The addition of a centrifugation step before extraction, to separate microsomes from supernatant, or replacement of sodium hydroxide with acetone, methanol, formic acid, hydrochloric acid, sulfuric acid or trifluroacetic acid, to terminate the reaction, were tested. Although some of these modifications reduced the appearance of unknown non-interfering peaks in the blank samples, the modifications reduced the recovery of the $\mathrm{OH}-\mathrm{BDE}$ standards and were not incorporated into the assay.

\section{Method Validation}

Visual inspection of chromatograms obtained from blank samples and chromatograms obtained from spiked CS samples showed no interfering peaks at the same $\mathrm{m} / \mathrm{z}$ and retention times as the OH-BDE standards or internal standard. Representative chromatograms obtained from a CS sample at $50 \mathrm{nM}$ and a blank sample are shown in Figure 2.

Mean $\mathrm{R}^{2}$ values of the calibration curve constructed for each individual metabolite were $0.95 \pm 0.06$ for $4^{\prime}-$ OH-BDE-17, $0.96 \pm 0.04$ for 2'-OH-BDE-28, $0.94 \pm 0.05$ for $4-\mathrm{OH}-\mathrm{BDE}-42,0.96 \pm 0.04$ for $3-\mathrm{OH}-\mathrm{BDE}-47,0.97$ \pm 0.04 for 5-OH-BDE-47, $0.94 \pm 0.06$ for $6-\mathrm{OH}-\mathrm{BDE}-47$ and $0.95 \pm 0.05$ for 4 '-OH-BDE-49. The LOQ concentration of individual $\mathrm{OH}-\mathrm{PBDEs}$ ranged between 5 and 50 nM (Table 1). The LOQ concentrations determined in this study, which are in the $\mathrm{pg} / \mathrm{mL}$ range, are similar to those reported by Erratico, et al., for OH-penta-BDEs using a similar UPLC/MS method [28] and comparable to those reported by Mas, et al. [24], using LC/MS ion spray operated in negative ion MRM mode.

Intra- and inter-day accuracy and precision values are reported in Tables 2 and 3, respectively. Both intraand inter-day accuracy and precision fell within the established acceptance criteria. In contrast to the study by Mas, et al. [24], our experimental design evaluated ac-

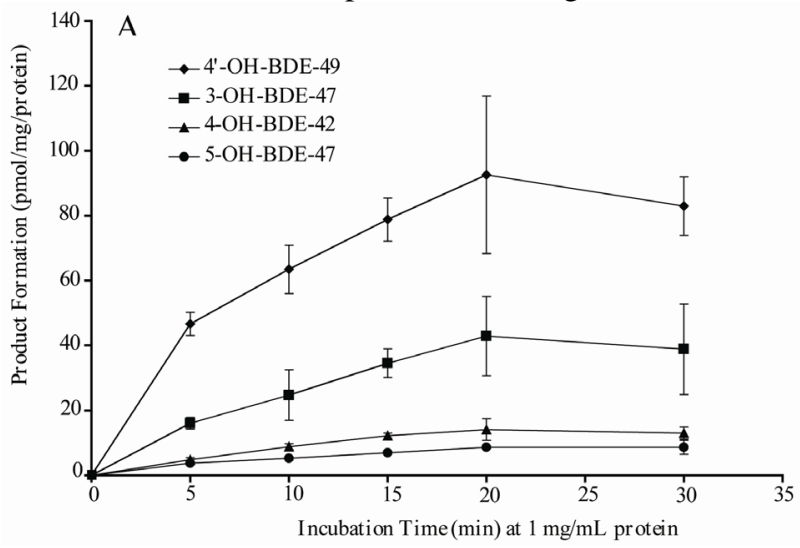

(a)

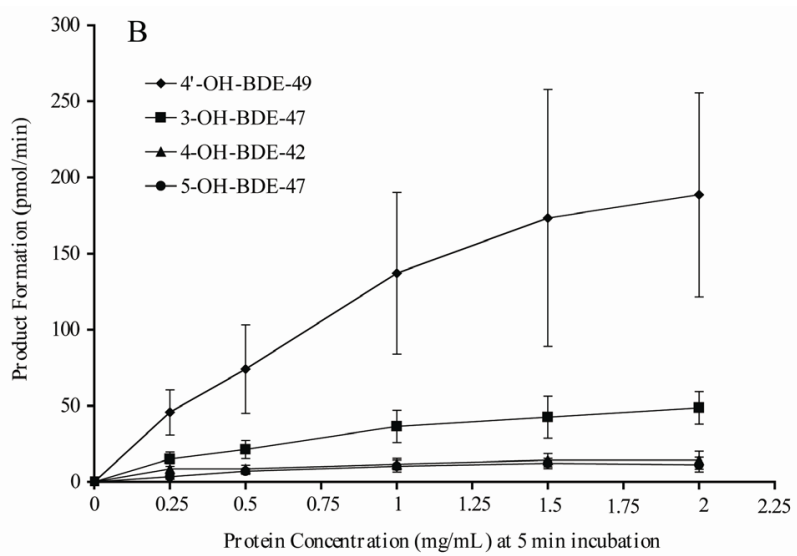

(b)

Figure 3. Effect of (a) incubation time and (b) microsomal protein concentration on OH-BDE metabolite formation. BDE-47 (50 $\mu \mathrm{M})$ was incubated with hepatic microsomes prepared from phenobarbital-treated adult male rats for (a) 0-30 $\mathrm{min}$ at $1 \mathrm{mg}$ microsomal protein/mL or (b) $5 \mathrm{~min}$ at 0 - 2 mg microsomal protein/mL. OH-BDE metabolites were extracted and analyzed by UPLC/MS as described in the Experimental section. Data points are mean \pm SEM.

curacy and precision using the biological matrix of interest and concentrations at the low, medium and high range of the calibration curve rather than at a single point in an organic solvent (i.e., $75 \mathrm{pg} / \mathrm{ml}$, approximately 150 $\mathrm{nM}$ ). Evaluation using the biological matrix and multiple points on the calibration curve provides a more robust estimation of accuracy and precision. 
Recovery values for the low and medium QC sample ranged between $84 \%$ and $89 \%$ for all OH-BDEs. Recovery values for the high QC sample were slightly lower, ranging between $70 \%$ and $77 \%$ (Table 4 ). The recovery values are higher than those reported in a previous study that used GC-based methods [19], but lower than those reported by Erratico, et al. [28] using a similar method to identify the oxidative metabolites of BDE-99. Increasing the extraction volume or the number of extractions, did not increase recovery rates of the OH-PBDE standards.

The method validation studies confirm that OH-BDEs are amenable to direct analysis by UPLC/MS in negative electrospray ion mode without the need for derivatization or extensive fragmentation and that the UPLC/MS-based analytical method that we developed can be used for the separation, detection and quantification of OH-BDEs in a complex biological matrix such as rat liver microsomes.

\subsection{Biotransformation of BDE-47 by Rat Liver Microsomes}

The validated UPLC/MS method was applied to analyze the oxidative metabolism of BDE-47 in vitro. Incubation of BDE-47 with rat hepatic microsomes prepared from PB-treated adult male rats produced five $\mathrm{OH}$-tetra-BDE metabolites (4-OH-BDE-42, 3-OH-BDE-47, 5-OH-BDE47, 6-OH-BDE-47 and 4'-OH-BDE-49) which were detected and identified by their retention times and $\mathrm{m} / \mathrm{z}$ values. The major metabolite was 4'-OH-BDE-49. There was no evidence for the formation of 4'-OH-BDE-17 or 2'- OH-BDE-28 by hepatic microsomes prepared from PB- treated rats. No other metabolite or unidentified peaks were observed.

The effect of varying incubation time $(0-30 \mathrm{~min})$ and protein concentration $(0-2 \mathrm{mg} / \mathrm{mL})$ on formation of the five hydroxylated metabolites was investigated using a substrate (BDE-47) concentration of $50 \mu \mathrm{M}$. Formation of 4-OH-BDE-42, 3-OH-BDE-47, 5-OH-BDE-47 and 4'OH-BDE-49 was linear for the first 5 min of incubation at a microsomal protein concentration of $1 \mathrm{mg} / \mathrm{mL}$ (Figure 3a) and approximately linear up to a microsomal protein concentration of $1 \mathrm{mg} / \mathrm{mL}$ at an incubation time of 5 min (Figure 3b). Under the experimental conditions used, formation of 6-OH-BDE-47 was detected but could not be quantified because the concentration of this metabolite remained below the LOQ value $(10 \mathrm{nM})$.

\section{Conclusions}

A UPLC/MS-based analytical method for the separation, detection and quantification of seven possible oxidative metabolites of BDE-47 in rat liver microsomes was developed and validated. The method was applied to ana- lyze the oxidative metabolism of BDE-47 in vitro and allowed us to detect formation of five monohydroxylated metabolites of BDE-47 and quantify formation of four of the metabolites. Our method represents an improvement of previously published LC/MS methods because validation was performed using the biological matrix of interest and at the low, medium and high ends of the calibration curve providing a more complete assessment of the accuracy, precision, recovery and LOQ of the analytical method. Quantification of hydroxylated metabolites of BDE-47 generated by hepatic microsomes following a 5 min incubation at $1 \mathrm{mg}$ microsomal protein/mL demonstrates the sensitivity and feasibility of the method for further in vitro metabolic studies, including reaction phenotyping, analyzing enzyme-catalyzed reaction kinetics and enzyme inhibition. The selectivity and reproducibility of the UPLC/MS method combined with the in vitro metabolism assay will be useful for measuring metabolism of BDE-47 in human liver samples and in liver preparations from additional species.

\section{References}

[1] P. Vonderheide, K. E. Mueller, J. Meija and G. L. Welsh, "Polybrominated Diphenyl Ethers: Causes for Concern and Knowledge Gaps Regarding Environmental Distribution, Fate and Toxicity," Science of the Total Environment, Vol. 400, No. 1-3, 2008, pp. 425-436. doi:10.1016/j.scitotenv.2008.05.003

[2] M. Alaee, P. Arias, A. Sjödin and Å. Bergman, “An Overview of Commercially Used Brominated Flame Retardants, Their Applications, Their Use Patterns in Different Countries/Regions and Possible Modes of Release,” Environment International, Vol. 29, No. 6, 2003, pp. 683-689. doi:10.1016/S0160-4120(03)00121-1

[3] R. C. Hale, M. Alaee, J. B. Manchester-Neesvig, H. M. Stapleton and M. G. Ikonomou, "Polybrominated Diphenyl Ether Flame Retardants in the North American Environment," Environment International, Vol. 29, No. 6, 2003, pp. 771-779. doi:10.1016/S0160-4120(03)00113-2

[4] M. J. La Guardia, R. C. Hale and E. Harvey, "Detailed Polybrominated Diphenyl Ether (PBDE) Congener Composition of the Widely Used Penta-, Octa-, and Deca-PBDE Technical Fame-Retardant Mixtures,” Environmental Science and Technology, Vol. 40, No. 20, 2006, pp. 6247-6254. doi:10.1021/es060630m

[5] R. C. Hale, M. J. La Guardia, E. Harvey and T. M. Mainor, "Potential Role of Fire Retardant-Treated PolyureThane Foam as a Source of Brominated Diphenyl Ethers to the US Environment," Chemosphere, Vol. 46, No. 5, 2002, pp. 729-735. doi:10.1016/S0045-6535(01)00237-5

[6] F. Rahman, K. H. Langford, M. D. Scrimshaw and J. N. Lester, "Polybrominated Diphenyl Ether (PBDE) Flame Retardants,” Science of the Total Environment, Vol. 275, No. 1-3, 2001, pp. 1-17. 


\section{doi:10.1016/S0048-9697(01)00852-X}

[7] H. M. Stapleton, A. Sjödin, R. S. Jones, S. Niehüser, Y. Zhang and D. G. Patterson, "Serum Levels of PolybroMinated Diphenyl Ethers (PBDEs) in Foam Recyclers and Carpet Installers Working in the United States," Environmental Science and Technology, Vol. 42, No. 9, 2008, pp. 3453-3458. doi:10.1021/es7028813

[8] D. O. Odusanya, J. O. Okonkwo, and B. Botha, "PolyBrominated Diphenyl Ethers (PBDEs) in Leachates From Selected Landfill Sites in South Africa," Waste Management, Vol. 29, No. 1, 2009, pp. 96-102.

[9] M. Noël, N. Dangerfield, R. A. S. Hourston, W. Belzer, P. Shaw, M. B. Yunker and P. S. Ross. "Do trans-Pacific Air Masses Deliver PBDEs to Coastal British Columbia, Canada?” Environmental Pollution, Vol. 157, No. 12, 2009, pp. 3404-3412. doi:10.1016/j.envpol.2009.06.025

[10] K. Law, T. Halldorson, R. Danell, G. Stern, S. Gewurtz, M. Alaee, C. Marvin, M. Whittle, and G. Tomy, "BioacCumulation and Trophic Transfer of Some Brominated Flame Retardants in a Lake Winnipeg (Canada) Food Web," Environmental Toxicology and Chemistry, Vol. 25, No. 8, 2006, pp. 2177-2186. doi:10.1897/05-500R.1

[11] N. G. Dodder, B. Strandberg and R. A. Hites, “Concentrations and Spatial Variations of Polybrominated Diphenyl Ethers and Several Organochlorine Compounds in Fishes From the Northeastern United States," Environmental Science and Technology, Vol. 36, No. 2, 2002, pp. 146-151. doi:10.1021/es010947g

[12] M. A. McKinney, I. Stirling, N. J. Lunn, E. Peacock and R. J. Letcher, "The Role of Diet on Long-Term Concentration and Pattern Trends of Brominated and Chlorinated Contaminants in Western Hudson Bay Polar Bears, 1991-2007," The Science of the Total Environment, Vol. 408, No. 24, 2010, pp. 6210-6222. doi:10.1016/j.scitotenv.2010.08.033

[13] C. Kelly, M. G. Ikonomou, J. D. Blair and F.A.P.C. Gobas, "Hydroxylated and Methoxylated Polybrominated Diphenyl Ethers in a Canadian Arctic Marine Food Web," Environmental Science and Technology, Vol. 42, No. 19, 2008, pp. 7069-7077. doi:10.1021/es801275d

[14] A. Mazdai, N. G. Dodder, M. P. Abernathy, R. A. Hites and R. M. Bigsby, "Polybrominated Diphenyl Ethers in Maternal and Fetal Blood Samples," Environmental Health Perspectives, Vol. 111, No. 9, 2003, pp. 12491252. doi:10.1289/ehp.6146

[15] B. Johnson-Restrepo, K. Kannan, D.P. Rapaport and B.D. Rodan, "Polybrominated Diphenyl Ethers and PolyChlorinated Biphenyls in Human Adipose Tissue from New York," Environmental Science and Technology, Vol. 39, No. 14, 2005, pp. 5177-5182. doi:10.1021/es050399x

[16] A. Schecter, M. Pavuk, O. Päpke, J. J. Ryan, L. Birnbaum and R. Rosen, "Polybrominated Diphenyl Ethers (PBDEs) in U.S. Mothers' Milk,” Environmental Health Perspectives, Vol. 111, No. 14, 2003, pp. 1723-1729. doi:10.1289/ehp.6466

[17] J. R. Gee and V. C. Moser, “Acute Postnatal Exposure to Brominated Diphenylether 47 Delays Neuromotor Ontogeny and Alters Motor Activity in Mice,” Neurotoxicol- ogy and Treatment, Vol. 30, No. 2, 2008, pp. 79-87.

[18] C. E. Talsness, S. N. Kuriyama, A. Sterner-Kock, P. Schnitker, S. W. Grande, M. Shakibaei, A. Andrade, K. Grote and I. Chahoud, "In Utero and Lactational Exposures to Low Doses of Polybrominated Diphenyl Ether47 Alter the Reproductive System and Thyroid Gland of Female Rat Offspring,” Environmental Health Perspectives, Vol. 116, No. 3, 2008, pp. 308-314. doi:10.1289/ehp.10536

[19] T. Hamers, J. H. Kamstra, E. Sonneveld, A. J. Murk, T. J. Visser, M. J. M. Van Velzen, A. Brouwer and Å. Bergman, "Biotransformation of Brominated Flame Retardants into Potentially Endocrine-Disrupting Metabolites, with Special Attention to 2,2',4,4'-Tetrabromodiphenyl ether (BDE-47)," Molecular Nutrition \& Food Research, Vol. 52, No. 2, 2008, pp. 284-298. doi:10.1289/ehp.10536

[20] J. M. Sanders, L.-J. Chen, E. H. Lebetkin and L. T. Bur$\mathrm{ka}$ "Metabolism and Disposition of 2,2',4,4'-Tetrabromodi- phenyl Ether Following Administration of Single or Multiple Doses to Rats and Mice," Xenobiotica, Vol. 36, No. 1, 2006, pp. 103-117. doi:10.1080/00498250500485107

[21] G. Marsh, M. Athanasiadou, I. Athanassiadis and A. Sandholm, "Identification of Hydroxylated Metabolites in 2,2',4,4'-Tetrabromodiphenyl Ether Exposed Rats," Chemosphere, Vol. 63, No. 4, 2006, pp. 690-697. doi:10.1016/j.chemosphere.2005.07.072

[22] S. W. Cheng, K. Randal and, A. T. Kotchevar. "In Vitro Metabolism Studies of Polybrominated Diphenyl Ethers using Rat and Human Liver Microsomes,” American Journal of Biochemistry and Biotechnology, Vol. 4, No. 3, 2008, pp. 295-303. doi:10.3844/ajbbsp.2008.295.303

[23] M. Athanasiadou, S. N. Cuadra, G. Marsh, Å. Bergman and K. Jakobsson, "Polybrominated Diphenyl Ethers (PBDEs) and Bioaccumulative Hydroxylated PBDE Metabolites in Young Humans from Managua, Nicaragua," Environmental Health Perspectives, Vol. 116, No. 3, 2008, pp. 400-408. doi:10.1289/ehp.10713

[24] S. Mas, O. Jauregui, F. Rubio, A. de Juan, R. Tauler and S. Lacorte, "Comprehensive Liquid Chromatography-Ionspray Tandem Mass Spectrometry Method for the Identification and Quantification of Eight Hydroxylated Brominated Diphenyl Ethers in Environmental Matrices," Journal of Mass Spectrometry, Vol. 42, No. 7, 2007, pp. 890-900. doi:10.1002/jms.1224

[25] P. R. Edwards, E. G. Hrycay and S.M. Bandiera, "DifFerential Inhibition of Hepatic Microsomal AlkoxyreSorufin O-Dealkylation Activities by Tetrachlorobiphenyls," Chemico-Biological Interactions, Vol. 169, No. 1 2007, pp. 42-52. doi:10.1016/j.cbi.2007.05.004

[26] T. Omura and R. Sato, “The Carbon Monoxide-Binding Pigment of Liver Microsomes I: Evidence for its Hemoprotein Nature," The Journal of Biological Chemistry, Vol. 239, No. 7, 1964, pp. 2370-2378.

[27] O. H. Lowry, N. J. Rosebrough, A. L. Farr and R. J. Randall, "Protein Measurement with the Folin Phenol Reagent,” The Journal of Biological Chemistry, Vol. 193, 1951, pp. 265-275. 
[28] A. Erratico, A. Szeitz and S. M. Bandiera. "Validation of a Novel in Vitro Assay Using Ultra Performance Liquid Chromatography-Mass Spectrometry (UPLC/MS) to Detect and Quantify Hydroxylated Metabolites of
BDE-99 in Rat Liver Microsomes,” Journal of chromatography B, Analytical technologies in the biomedical and life sciences, Vol. 878, No. 19, 2010, pp. 1562-1568. doi:10.1016/j.jchromb.2010.04.014 